\title{
Mathematical Modelling of the Transmission Dynamics of Ebola Virus
}

\author{
Amenaghawon C. Osemwinyen ${ }^{1,}$, Aboubakary Diakhaby ${ }^{2}$ \\ ${ }^{1}$ Department of Mathematics, FCT-College of Education, Zuba-Abuja, Nigeria \\ ${ }^{2}$ Department of Mathematics, Gaston Berger University, Saint-Louis, Senegal \\ Email address: \\ amenosem@yahoo.ca (A. C. Osemwinyen), diakhaby81@yahoo.fr (A. Diakhaby)
}

\section{To cite this article:}

Amenaghawon C. Osemwinyen, Aboubakary Diakhaby. Mathematical Modelling of the Transmission Dynamics of Ebola Virus. Applied and Computational Mathematics. Vol. 4, No. 4, 2015, pp. 313-320. doi: 10.11648/j.acm.20150404.19

\begin{abstract}
The study simulated the transmission dynamics of Ebola Zaire virus using two models: a modified SIR model with the understanding that the recovered can become infected again and the infected die at a certain rate and a quarantine model, which ascertained the effects of quarantining the infected. Furthermore, an appropriate system of Ordinary Differential Equations (ODE) was formulated for the transmission and the method of linearized stability approach was used to solve the equations. Stability analysis of both models indicated that, the Disease Free Equilibrium (DFE) states of the models were unstable if they exist. These equilibria states showed that the disease can easily be triggered off, so there is need to be constantly alert and effective preventive measures put in place against its spread. In addition, numerical experiments were carried out with the models' parameters assigned specific hypothetical values and graphs were plotted to investigate the effect of these parameters on the transmission of the disease. The results showed that, with the nature of Ebola Zaire virus, uncontrolled transmittable contacts between the infected and the susceptible can lead to a very serious outbreak with high mortality rate, since no immunity and drugs at moment. However, with effective quarantining structures put in place such situation can be better managed and outbreak controlled.
\end{abstract}

Keywords: Mathematical Model, Ebola Virus, Transmission Dynamics

\section{Introduction}

The Ebola virus is a virological taxon species of the genus Ebolavirus. The genus Ebolavirus belongs to the virus family Filoviridae (filovirus) along side with genus Marburgvirus and genus Cuevavirus [1]. Genus Ebolavirus comprises five distinct species according to World Health Organisation (WHO): Bundibugyo ebolavirus (BDBV); Zaire ebolavirus (EBOV); Reston ebolavirus (RESTV); Sudan ebolavirus (SUDV) and Taï Forest ebolavirus (TAFV)

The Ebola virus or Zaire ebolavirus (EBOV) specie which first appeared in 1976 in Yambuku, Democratic Republic of Congo is a deadly viral that causes an extremely severe hemorrhagic fever in humans and other primates [2]. This Ebola Hemorrhagic Fever (EHF) is characterized by high fever, vomiting, diarrhoea and in severe cases, organ failure and unstoppable bleeding (virus shutting off the blood's ability to clot) and as a result, patients often suffer internal and external hemorrhaging. Many die in an average of 10 days [3]. The disease can cause mortality rates up to 90 percent of those individuals who contract the disease [4]. So far, no cure or vaccine exists for Ebola Hemorrhagic Fever and over 10,994 people [5] in West Africa have died in the current and worst Ebola outbreak which started in Guinea sometime in December 2013, but was not detected until March 2014, [6] after which it spread to Liberia, Sierra Leone, Nigeria and Senegal.

The virus' exact origin, location and natural habitat is considered to be within the region's fruit bat population, with the fruit bats (of the Pteropodidae family) carriers of Ebola, but unaffected by it [7]. Although, non-human primates have been a source of infection for humans, they are not thought to be the reservoir but rather an accidental host like human beings [4]. Humans can catch the virus from animals through close contact with infected animals' blood, secretions, organs or other bodily fluids, although transmission between natural reservoirs and humans is rare. Outbreaks are often traceable to a single index case where an individual has handled the 
carcass of a gorilla, chimpanzee, or duiker or the bushmeat trade (the catching and eating of wild animals, including primates such as gorillas and chimpanzees) [8]. Once in the human population, transmission through physical contact with body fluids, secretions, tissues or semen from infected persons becomes highly possible especially within families, hospitals, and during some mortuary rituals where contact among individuals becomes more likely.

These mortuary rituals, which seem to be a common practice within the West Africa sub-region, is one of the factors affecting the fight against the disease. Others include; the uncooperative nature of the citizens (believing the disease is a hoax or that the hospitals are responsible for the disease); traditional/cultural practice regarding the deceased i.e watching of dead bodies during burial rituals; extreme poverty and dysfunctional healthcare system. These factors and others have lead governments of these countries to embark on the following steps in order to reduce its spread: public awareness against burial rituals; banning of large gatherings, closing of borders; increased surveillance at all entry points to the country; conducting tests on people arriving in the country; placing worst affected areas under quarantine etc. Assistances in the form of medical staff support and funds have been given to some of the countries in order to strenghten the fight against the disease: Economic Community of West African States (ECOWAS), US Centers for Disease Control and Prevention (CDC) and European Commission have donated funds and mobilised personnel to help counter the outbreak. Charities including Médecins Sans Frontières, the Red Cross [9] and Samaritan's Purse are also working in some of these areas.

This outbreak has had significant economic impacts on the people apart from the loss of lives. Markets and shops are closed due to travel restrictions, cordon sanitaire, or fear of human contact leading to loss of income for producers and traders; adverse effects on tourism and reduced airline traffic leading to some airlines suspending flights to these areas. Others are; reduced agricultural activities and direct/indirect strain on finances of governments of some affected countries e.g, Sierra Leone using Treasury bills to fund the fight against the virus, etc.

By nature, Ebola is highly contagious and deadly and there are no drugs or proven Ebola virus-specific treatment at moment. Ebola can kill up to $90 \%$ of patients, although in this outbreak, the death rate has dropped below 50\%, which gives credence to the fact that early detection and good medical care could be synonymous to possible recovery, which is not permanent since with no immunity, the recovered become susceptible again. WHO estimated about 20,000 possible total cases for this outbreak but currently there are over 26,000 reported cases of which over 10,994 are dead. This is the first Ebola outbreak to reach epidemic proportions: past outbreaks were brought under control within a few weeks [5].

In the light of the foregoing, there is therefore an urgent and serious need to have coordinated responses from all angles in other to combat the disease effectively. As part of this coordinated approach, is the need for mathematical modelling of the disease. According to [10], a mathematical model is an equation, or set of equations, that describes some physical problem or phenomenon that has its origin in science, engineering, or some other areas while mathematical modelling is the process by which we obtain and analyze the model. Mathematical modelling of diseases is essential in epidemiology and some infectious diseases such as measles, malaria, chickenpox, aides and gonorrhea have been modelled in the past [11]. Others also modelled include SARS, avian flu and HIV [12].

Mathematical models have been important tools in analyzing the epidemiological characteristics of these infectious diseases since the pioneer work of Kermack and McKendrick (1927) [13]. Some of the well-known models for the transmission dynamics of some diseases include: Ronald Ross model for control of malaria [12]; Capasso and Pareri-Fontana (1979) model for the 1973 cholera epidemic [14]; Hethcote and Yorke (1984) model for the spread and control of gonorrhea [14] etc.

This work will therefore model the dynamics: transmission, severity and prevention mechanism of Ebola-Zaire strain (Zaire ebolavirus (EBOV)). This strain has had the most outbreaks of the four strains that cause disease in humans and it is the deadliest. It is responsible for the current outbreak in Guinea, Sierra Leone, Liberia, Nigeria, Mali, Senegal, Spain and United States where over 26,000 cases and 10,994 deaths have been reported [1]. This Ebola-Zaire strain, according to [8,5] has had an overall survival rate of roughly $30 \%$ and $70 \%$ mortality rate since it was first discovered in 1976.

Significanctly, the study will enlighten governments of various countries, International Organizations, NonGovernmental Organization (NGOs), health workers and the public on the transmission dynamics of the disease. More importantly the study will play a key role in policy making at different levels of governance as health workers will be steered up to urgently come up with a vaccine that will cure or reduce the spread of the disease having known the devastating effects of the disease. It will finally encourage governments of affected countries on the need for effective quarantining structures to enable control of the spread of the disease.

\section{Purpose of the Study}

The main focus of this study was to model mathematically the transmission dynamics of Ebola Zaire Virus and specifically:

1. To simulate the transmission dynamics of the disease using a modified SIR model of [2].

2. To refine this model to include a quarantined group and equally use it to simulate the transmission dynamics of the disease to ascertain the effects of quarantining

3. To investigate the existence and stability of the equilibrium states of the two models: modified SIR model and the model with quarantine.

4. To numerically analyze both models with the aim of using hypothetical data for predictions 


\section{Model Derivation}

\subsection{The Modified SIR Model}

This Modified SIR Model of [2] is different from a typical SIR model in two ways:

(i) This model will be a lethal i.e. fatal since there is no cure or immunity and the infected will die at a certain rate unlike the typical SIR model which is non-lethal.

(ii) The Recovered group will not remain immune to the infected unlike the general SIR model where the recovered group obtains immunity from the disease after they have been infected.

Other assumptions are:

- The total population of people used in this model is randomly distributed over area, allowing a constant to be defined for the contact made between the susceptible and the infected.

- The virus always kills about $\delta$ percent of the people it infects, the survivors $(100-\delta)$ percent will become the recovered group.

- Individuals that recover are given no immunity.

- The population involved stays constant (no births or unrelated deaths).

Based on the above, we consider four classes:

- Susceptible:(S)

- Infected:(I)

- Recovered:(R)

- Deceased:(D)

The susceptible (S) becomes infected at rate " $\alpha$ " when they come in contact with the infected so there will be depletion of the population. That is, the change in population is equal to $-\alpha$ SI. In addition, individuals from the recovered group become susceptible again at a certain rate " $\gamma$ ", to give $\gamma$ R. Thus, we have:

$$
S^{\prime}=-\alpha S I+\gamma R
$$

The infected group begins with adding what was just removed from the susceptible population, $\alpha \mathrm{SI}$ and then a reduction in two ways i.e. people can either recover or they are killed by the virus. They recovered from the virus at rate " $\beta$ " and are killed at rate " $\delta$ ". Thus, we have:

$$
I^{\prime}=\alpha S I-\beta I-\delta I
$$

The recovered group is increased by those that recovered from the virus and reduced by the number of people that join the susceptible group at rate " $\gamma$ ". This can be expressed as:

$$
R^{\prime}=\beta I-\gamma R
$$

While the deceased group is the final group. Its population is defined by the number of people that are killed by the virus at rate " $\delta$ ". Thus, the equation is given as:

$$
D^{\prime}=\delta I
$$

Therefore, the modified model comprises of the following system of differential equations:

$$
\left\{\begin{array}{c}
S^{\prime}=-\alpha S I+\gamma R \\
I^{\prime}=\alpha S I-\beta I-\delta I \\
R^{\prime}=\beta I-\gamma R \\
D^{\prime}=\delta I
\end{array}\right.
$$

Where $\alpha=$ the rate of infection, $\gamma=$ the rate of susceptibility

$\beta=$ the rate of recovery $\delta=$ the rate of death

$$
S^{\prime}=\frac{d S(t)}{d t}, I^{\prime}=\frac{d I(t)}{d t}, R^{\prime}=\frac{d R(t)}{d t} \text { and } D^{\prime}=\frac{d D(t)}{d t}
$$

Here, it is important to note that, the dependent variables: S, I, R and D count people in each group as a function of time and not representing each group by a fraction of the population. Therefore, the transmission process used is the mass action transmission and the transmission process for the nonlinear term $\alpha \mathrm{SI}$ is given as:

$$
\alpha N \times \frac{S}{N} \times I=\alpha S I
$$

Where, $\mathrm{N}=$ Total number of Population,

$\alpha N=$ number of contacts by the infected per unit time sufficient to transmit the disease.

$\frac{S}{N}$ is the fraction of these contacts that are with the susceptible.

The model is illustrated below:

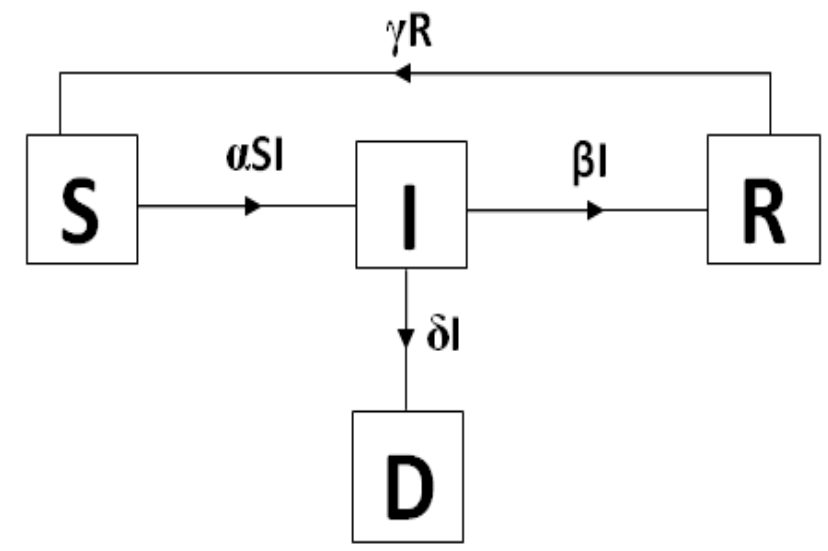

Figure 1. The SIRD model flow chart: Basic model with death and the recovered becoming susceptible.

\subsection{The Model with Quarantine}

In order to contain the outbreak, we decided to model the effects of partial quarantine of the infected individuals. In this model, we assume that quarantined individuals are removed from the population and cannot infect new individuals while they remain quarantined. Thus, the changes to the modified model include:

- The quarantined area only contains members of the infected population (entering at rate $\omega$ )

- The early detected infected individuals may have temporal recovery after treatment and be moved to the recovered group at rate $\beta$.

- Those not discovered early or failed to respond to 
treatment may die and be moved to the deceased group at rate $\delta$.

Therefore, the model equations are:

$$
\left\{\begin{array}{c}
S^{\prime}=-\alpha S I+\gamma R \\
I^{\prime}=\alpha S I-\omega I \\
Q^{\prime}=\omega I-\beta Q-\delta Q \\
R^{\prime}=\beta Q-\gamma R \\
D^{\prime}=\delta Q
\end{array}\right.
$$

Where

$\alpha=$ rate of infection $\beta=$ rate of recovery

$\gamma=$ rate of susceptibility $\omega=$ Rate of quarantining

$\delta=$ Rate of death

$$
S^{\prime}=\frac{d S(t)}{d t}, I^{\prime}=\frac{d I(t)}{d t}, Q^{\prime}=\frac{d Q(t)}{d t}, R^{\prime}=\frac{d R(t)}{d t}
$$

and

$$
D^{\prime}=\frac{d D(t)}{d t}
$$

The model is illustrated in the figure below:

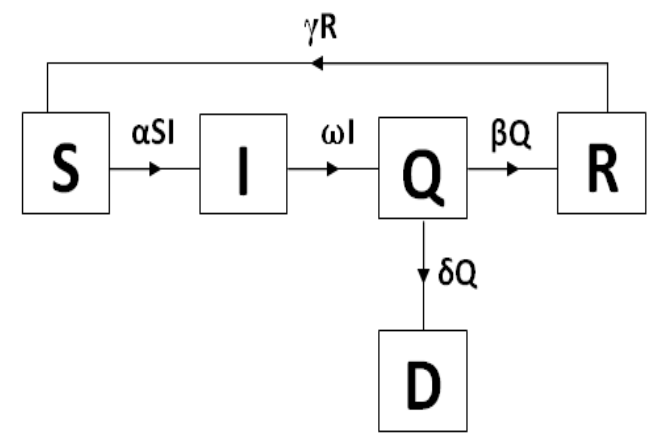

Figure 2. Model flow diagram for the Model with Quarantine.

$$
\left|f\left(x_{1}, t\right)-f\left(x_{2}, t\right)\right| \leq M\left|x_{1}-x_{2}\right|
$$

$\forall\left(\mathrm{x}_{1}, \mathrm{t}\right),\left(\mathrm{x}_{2}, \mathrm{t}\right) \in \mathrm{U} \times[0, \mathrm{~T}]$

(where $\mathrm{M}$ is a given constant). If $\mathrm{x}_{0} \in \mathrm{U}$, then for some positive $\delta$ there is a unique solution $\mathrm{x}:[0, \delta] \rightarrow \mathrm{U}$ of the initial value problem (4). A similar statement holds if $[0, \mathrm{~T}]$ is replaced by $[-\mathrm{T}, 0]$ or $[-\mathrm{T}, \mathrm{T}]$ (the interval of existence becomes then, respectively, $[-\delta, 0]$ and $[-\delta, \delta])$.

Theorem 4.1.4: According to [11]: Suppose the Jacobian Matrix $J$ corresponding to an n-system of differential equations has eigenvalues $\lambda_{1}, \lambda_{2}, \ldots, \lambda_{n}$, for a trivial steady state equilibrium at $(0,0, \ldots, 0)$, then the stability of the solution is determined as follows:

(i) All real parts are less than zero i.e. if $\operatorname{Re}\left(\lambda_{i}\right)<0$ for all $i=1,2, \ldots n$ then there is Uniform and Asymptotic Stability (UAS).

(ii) If $\operatorname{Re}\left(\lambda_{i}\right) \leq 0$ for all $i=1,2, \ldots . n$ and the algebraic multiplicity equals the geometric multiplicity, whenever $\lambda_{i}=0$ for any $i$ then there is Uniform Stability (US).

(iii) At least one of the eigen values has real part greater than 0 i.e. if $\lambda_{i}>0$ for at least one $i$ and the algebraic multiplicity is greater than the geometric multiplicity whenever $\lambda_{i}=0$ for any $i$, then there is

\section{Model Analysis}

\subsection{Existence and Uniqueness Theorems of First Order ODE}

In this section, the following theorems will be relevant: Given the general first-order ODE:

$$
\mathrm{y}^{\prime}=\mathrm{F}(\mathrm{x}, \mathrm{y}) \quad \mathrm{y}\left(\mathrm{x}_{0}\right)=\mathrm{y}_{0}
$$

Theorem 4.1.1 (Existence): Suppose that $F(x, y)$ is a continuous function defined in some region $\mathrm{R}=$ $\left\{(\mathrm{x}, \mathrm{y}): \mathrm{x}_{0}-\delta<x<\mathrm{x}_{0}+\delta \quad, \quad \mathrm{y}_{0}-\varepsilon<y<\mathrm{y}_{0}+\varepsilon\right\}$ containing the point $\left(\mathrm{x}_{0}, \mathrm{y}_{0}\right)$. Then there exists a number $\delta_{1}$ (possibly smaller than $\delta$ ) so that a solution $\mathrm{y}=\mathrm{f}(\mathrm{x})$ to (3) is defined for $\mathrm{x}_{0}-\delta_{1}<x<\mathrm{x}_{0}+\delta_{1}$.

Theorem 4.1.2 (Uniqueness): Suppose that both $F(x, y)$ and $\frac{\partial}{\partial y} \mathrm{~F}(\mathrm{x}, \mathrm{y})$ are continuous functions defined on a region $\mathrm{R}$ as in Theorem 4.1.1. Then there exists a number $\delta_{2}$ (possibly smaller than $\delta_{1}$ ) so that the solution $y=f(x)$ to (3), whose existence was guaranteed by Theorem 4.1.1, is the unique solution to (3) for $\mathrm{x}_{0}-\delta_{2}<x<\mathrm{x}_{0}+\delta_{2}$.

Theorem 4.1.3 (Cauchy-Lipschitz theorem (Existence and Uniqueness of the solution)):

Given an initial value problem,

$$
\left\{\begin{array}{c}
x^{\prime}(t)=f(x(t), t) \\
x(0)=x_{0}
\end{array}\right.
$$

Let $\mathrm{U} \subset \mathrm{R}^{\mathrm{n}}$ be an open set and $\mathrm{f}: \mathrm{U} \times[0, \mathrm{~T}] \rightarrow \mathrm{R}^{\mathrm{n}}$ be a continuous function which satisfies the lipschitz condition

$$
\text { instability. }
$$

\subsection{Dynamics of the Models (Modified Sir Model and the Quarantine Model)}

A straight forward computation shows that models (1) \& (2) are continuous and Lipschizian in $\mathbb{R}_{+}^{3}$. From the existence and uniqueness of solution of the ordinary differential equation as contained in above theorems $(4.1 .1-4.1 .3)$, the solution of both models with positive initial conditions exists and are unique. Here, we can see for the first differential equation in both models, that it has a solution, which is also unique, i.e $S^{\prime}=-\alpha S I+\gamma R$ with positive initial conditions.

In this case, both the function $F(t, S)=-\alpha S I+\gamma R$ and its partial derivative $\frac{\partial}{\partial S} \mathrm{~F}(\mathrm{t}, \mathrm{S})=-\alpha \mathrm{I}$ are defined and continuous at all points $(\mathrm{t}, \mathrm{S})$. The theorem guarantees that a solution to the ODE exists in some open interval centered at $t_{0}$ and that this solution is unique in some (possibly smaller) interval centered at $t_{0}$.

NB: This is also true for the remaining equations in both models. 
We can also check for conformity with the Lipschitz condition:

Considering the first differential equation in both models,

$$
\text { i.e for } F(S, t)=-\alpha S I+\gamma R \text {, then, }\left|\mathrm{f}\left(\mathrm{s}_{1}, \mathrm{t}\right)-\mathrm{f}\left(\mathrm{s}_{2}, \mathrm{t}\right)\right| \leq M\left|S_{1}-S_{2}\right|, \forall\left(\mathrm{s}_{1}, \mathrm{t}\right),\left(\mathrm{s}_{2}, \mathrm{t}\right) \in \mathrm{U} \times[0, \mathrm{~T}]
$$

So we have,

$$
\begin{gathered}
\left|\left(-\alpha S_{1} I+\gamma R\right)-\left(-\alpha S_{2} I+\gamma R\right)\right|=\left|-\alpha S_{1} I+\gamma R+\alpha S_{2} I-\gamma R\right|=\left|-\alpha S_{1} I+\alpha S_{2} I\right|=\alpha I\left|(-1)\left(S_{1}-S_{2}\right)\right| \\
\leq \alpha I||-1\left|\left(S_{1}-S_{2}\right)\right| \leq \alpha I\left|S_{1}-S_{2}\right| \leq M\left|S_{1}-S_{2}\right|
\end{gathered}
$$

Where $M$ is a constant and $M=\alpha I$.

This shows the Lipschitz condition for the first differential equation in both models is true and is also true for the remaining equations in both models. So, solution exist for each model and it is unique.

\subsection{Existence and Stability of the Disease Free Equilibrium (DFE) of the Models}

\subsubsection{The Modified SIR Model}

At the equilibrium, the ODEs satisfy: $S^{\prime}+I^{\prime}+R^{\prime}+D^{\prime}=$ 0 , since $S+I+R+D=N$ and $N$ is constant (Here, we assume that the outbreak has a short timescale, then we can ignore birth and background death rates, i.e birth rate $=$ death rate $=0$ ). Equation (1) is then set to zero. Thus, we have

$$
\left\{\begin{array}{c}
-\alpha S I+\gamma R=0 \\
\alpha S I-\beta I-\delta I=0 \\
\beta I-\gamma R=0 \\
\delta I=0
\end{array}\right.
$$

Solving equation (6) above, we have the Disease Free Equilibrium (DFE) in the form:

$$
(\bar{S}, \bar{I}, \bar{R}, \bar{D})=\left(N-S_{0}, 0,0, S_{0}\right) \forall S_{0} \in R \cap\lfloor 0, N\rfloor
$$

Here, for instance the equilibrium $(\bar{S}, \bar{I}, \bar{R}, \bar{D})=$ $(N, 0,0,0)$ is of the above form where $S_{0}=0$ and this is dependent on the initial conditions.

In evaluating the stability of the Disease Free Equilibrium (DFE), we take the Jacobian Matrix of model (1). However, we notice that the differential equation in " $D$ " is uncoupled from the other three differential equations of the set and we also see that $\lim _{t \rightarrow \infty} I(t)=0$, since the population has a fixed number $N$. As such, the fourth equation can be derived from the first three. Thus, we consider the Jacobian for only the first three equations. That is,

$$
J(S, I, R)=\left[\begin{array}{ccc}
-\alpha I & -\alpha S & \gamma \\
\alpha I & \alpha S-\beta-\delta & 0 \\
0 & \beta & -\gamma
\end{array}\right]
$$

and the Jacobian at the DFE state is given as:

$$
J\left(N-S_{0}, O, O\right)=\left[\begin{array}{ccc}
0 & -\alpha\left(N-S_{0}\right) & \gamma \\
0 & \alpha\left(N-S_{0}\right)-\beta-\delta & 0 \\
0 & \beta & -\gamma
\end{array}\right]
$$

Furthermore, we have

$$
\operatorname{det}(J-\lambda I)=-\lambda\left((-\gamma-\lambda)\left(\alpha\left(N-S_{0}\right)-\beta-\delta-\lambda\right)\right)
$$

and equating our characteristic equation to zero to evaluate for $\lambda$, we have

$$
\begin{gathered}
\operatorname{det}(J-\lambda I)=0 \\
\text { i.e }-\lambda\left((-\gamma-\lambda)\left(\alpha\left(N-S_{0}\right)-\beta-\delta-\lambda\right)\right)=0
\end{gathered}
$$

Here, it follows that the characteristics equation always has a root with positive real part, that is, one of the eigenvalues has a real root $>0$ and this is true for all $S_{0} \in$ $R \cap[0, N]$. Hence, from theorem 4.1.4, the Disease-Free Equilibrium (DFE) is always unstable.

\subsubsection{The model with Quarantine}

At the equilibrium, the ODEs satisfy: $S^{\prime}+I^{\prime}+Q^{\prime}+R^{\prime}+$ $D^{\prime}=0$, since $S+I+Q+R+D=N$ and $N$ is constant. We then set (2) to zero. Thus, we have

$$
\left\{\begin{array}{c}
-\alpha S I+\gamma R=0 \\
\alpha S I-\omega I=0 \\
\omega I-\beta Q-\delta Q=0 \\
\beta Q-\gamma R=0 \\
\delta Q=0
\end{array}\right.
$$

Solving equation (7) above, we have the Disease Free Equilibrium (DFE) in the form:

$$
(\bar{S}, \bar{I}, \bar{Q}, \bar{R}, \bar{D})=\left(N-S_{0}, 0,0,0, S_{0}\right) \forall S_{0} \in R \cap[0, N]
$$

In evaluating the stability of the Disease Free Equilibrium (DFE), we take the Jacobian matrix of model (2). However, we notice also that the differential equation in " $D$ " is uncoupled from the other four differential equations of the set and that $\lim _{t \rightarrow \infty} I(t)=0$, since the population has a fixed number $N$. As such, the fifth equation can be derived from the first four. Thus, we consider the Jacobian for only the first four equations. That is,

$$
J(S, I, Q, R)=\left[\begin{array}{cccc}
-\alpha I & -\alpha S & 0 & \gamma \\
\alpha I & \alpha S-\omega & 0 & 0 \\
0 & \omega & -\beta-\delta & 0 \\
0 & 0 & \beta & -\gamma
\end{array}\right]
$$

and the Jacobian at the DFE state is given as:

$J\left(N-S_{0}, 0,0,0\right)=\left[\begin{array}{cccc}0 & -\alpha\left(N-S_{0}\right) & 0 & \gamma \\ 0 & \alpha\left(N-S_{0}\right)-\omega & 0 & 0 \\ 0 & \omega & -\beta-\delta & 0 \\ 0 & 0 & \beta & -\gamma\end{array}\right]$

Furthermore, we have $\operatorname{det}(J-\lambda I)=-\lambda\left(\left(\alpha\left(N-S_{0}\right)-\right.\right.$ $\omega-\lambda)(-\gamma-\lambda)(-\beta-\delta-\lambda))$ and equating our 
characteristic equation to zero to evaluate for $\lambda$, we have $-\lambda\left(\left(\alpha\left(N-S_{0}\right)-\omega-\lambda\right)(-\gamma-\lambda)(-\beta-\delta-\lambda)\right)=0$

It follows from above that the characteristics equation always has a root with positive real part, that is, one of the eigenvalues has a real root $>0$ and this is true for all $S_{0} \in$ $R \cap[0, N\rfloor$. Hence, from theorem 4.1.4, the Disease-Free Equilibrium (DFE) is always unstable. Thus, even at the DFE state the tendency for the disease to be triggered is there.

\section{Results}

\subsection{Numerical Experiments}

Euler's method with the aid of a Matlab program was used to approximate and simulate the solutions to the ODE's. With the obtained data, graphs were plotted to investigate the effect of some parameters on the transmission dynamics of the infection.

NB: Some of the parameters were computed as follows:

$\alpha=$ rate of infection: $\alpha=\mathrm{k} / \mathrm{N}$, where $\mathrm{k}=$ average number of adequate contacts made by an infected individual per time and $\mathrm{N}=$ total population size

$\delta=$ rate of death: approximated average mortality rate of the disease from 1976 before current outbreak and $\beta=$ rate of recovery $(1-\delta)$

\subsection{Experiment 1}

When there is high rate of transmittable contacts between infected and susceptibles: $\alpha=3.15 \times 10^{-5} ; \beta=0.27$; $\gamma=$ 0.23 and $\delta=0.73$

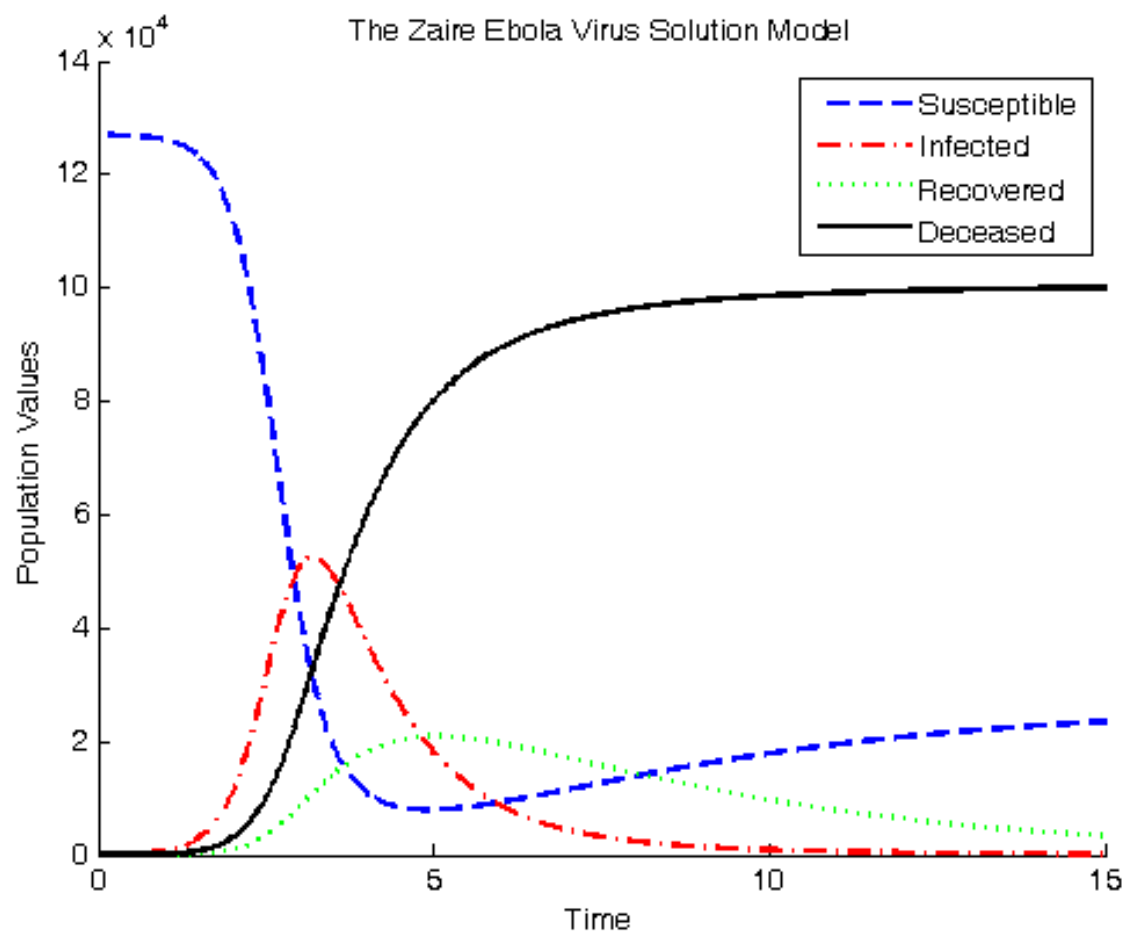

Figure 3. The Modified Model with relatively high contact.

From the graph (Fig. 3), we see a sudden drop of the susceptible group. The infected also begins to rise even to optimal point where about $39 \%$ of the population are infected within few days and then declines. The number of diseased also begins to rise due to the upward movement of the infected. The recovery group hits its peak at around 20,000 people but later begins to approach zero like the infected group even as the last victim either dies or joins the recovery group. Here, the virus took a very high percentage of the population say about $78 \%$. This could be horrific and portends a high epidemic situation but for the fact that Ebola virus spreads primarily through contacts and contaminated medical equipments and not an airborne disease or some other easily communicable disease, limits its ability to spread. Otherwise, this could spell doom.

\subsection{Experiment 2}

When transmittable contacts between infected and susceptibles is slightly reduced: $\alpha=2.36 \times 10^{-5} ; \beta=0.27 ; \gamma$ $=0.23$ and $\delta=0.73$

Fig. 4 shows relatively similar curves but with little improvement over Fig. 3 because of the relative decline in the number of contacts made by the infected group. Here, we have a gradual dropping of the susceptible group and a reduced peak of the infected (about $31 \%$ of population). The peak of the recovered group is not has high as in Fig. 3, however the deceased group is slightly lower than in Fig. 3, which is a mark of improvement. As the infected and recovery approached zero, susceptible increased higher as compared to Fig. 5 at the same stage. However, the death 
level is relatively still high, so a better option will be further reducing the contact of infected by quarantining, in addition

with medical support (see Fig. 5).

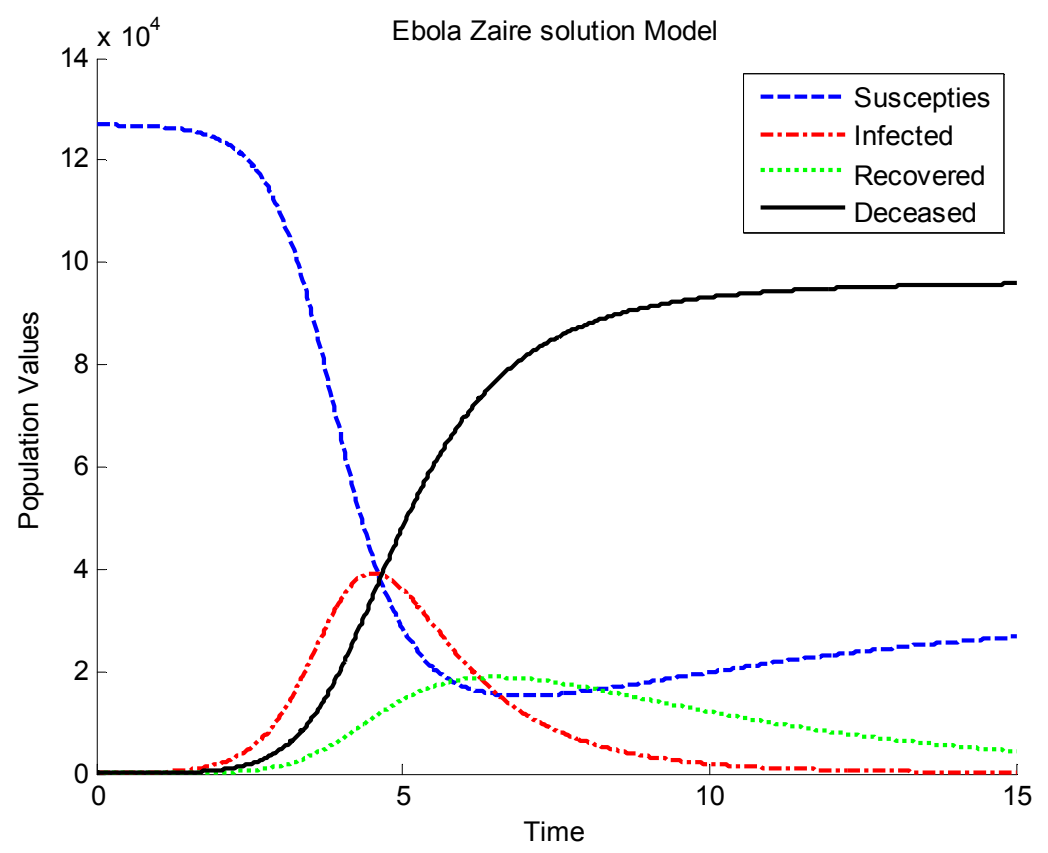

Figure 4. The Modified Model with slightly reduced contact.

\subsection{Experiment 3}

When infected are quarantined at relatively high rate i.e rate of transmittable contacts between infected and susceptibles is remarkably reduced: $\alpha=8.7 \times 10^{-6} ; \beta=0.27 ; \gamma=0.23 ; \omega=0.75$ and $\delta=0.73$

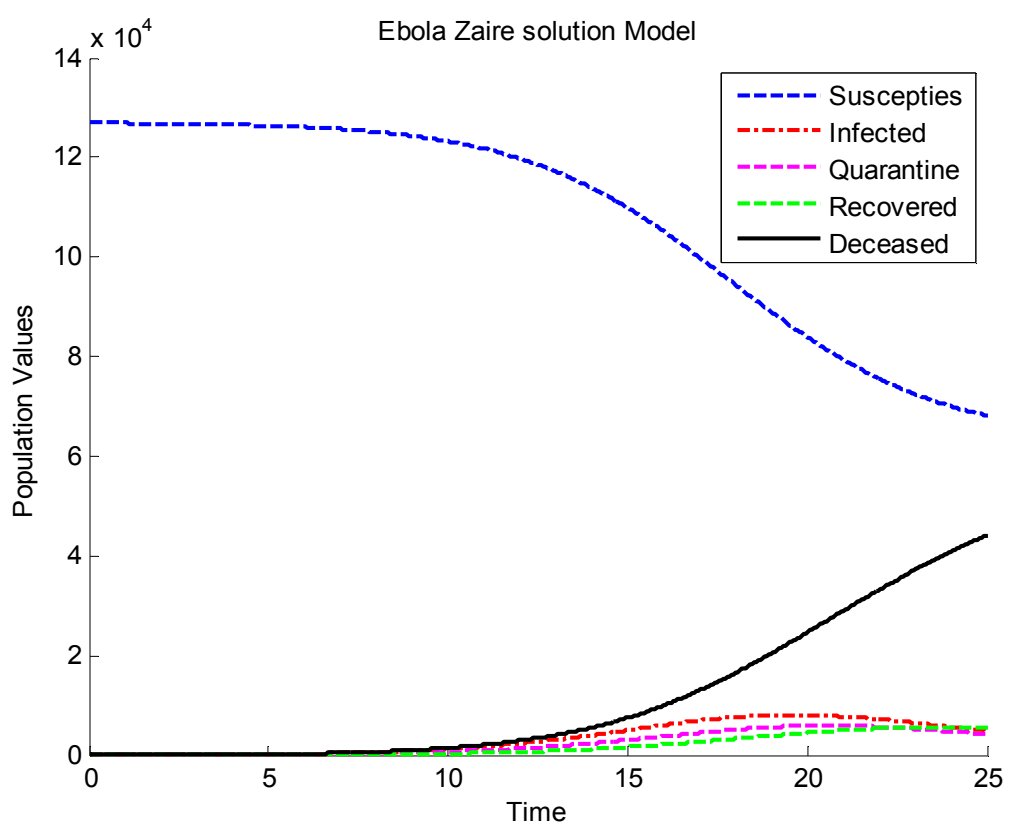

Figure 5. The Modified Model with Quarantine.

The result displayed in Fig. 5, shows a more interesting result than in Fig. 4 and Fig. 3. Here, the susceptible group is reducing very gradually and the infected group at its peak is quite low, about $6.3 \%$ of the population. At about that time, the quarantined group is a little lower in population than the infected. The recovered group is lower than both the infected and the quarantined and they all approach the zero mark together. The deceased group is relatively low as compared to 
Fig. 3 and Fig. 4 at about same time. Therefore, a greater percentage of the population is alive (i.e mortality rate is low), though still susceptible.

\section{Discussion}

Ebola virus by nature is very infectious and is fatal since there is no cure or immunity as of moment. From the numerical experiments carried out, it was observed firstly, that with high rate of transmittable contacts between infected and susceptible the effect was catastrophic. Here the death rate was very high, almost about $78 \%$ of the population died. Although this figure may be very different from reality but one incontestable fact is that, the level of fatality given any uncontrolled Ebola virus outbreak can always be worrisome. The experiments also revealed a situation where the contact was slightly reduced (from the previous high contact) in order to ascertain effects of varying the transmittable contact level and the result was similar to the previous one with slight reduction in the mortality level.

This in effect showed that the rate of contact is directly proportional to the mortality rate. Finally, the experiments also showed that the most likely way to curtail the spread and its fatal effects is the introduction of an aggressive quarantine system, which ensured very limited transmittable contacts between infected and susceptible. Although, aggressive quarantine system may sometimes be difficult in practice, due to some prevailing factors: economical, social or political but it is still the best option to be considered in managing the high fatality rate of Ebola outbreak at least with the no drugs or vaccine situation.

Furthermore, these results assumed that the timescale of the outbreak was short, so that, the natural birth and death rates could be ignored, making population $(\mathrm{N})$ to be constant.

\section{Conclusions}

An Ebola outbreak mostly in developing nations could be a real threat to peaceful existence. As demonstrated by our mathematical models it is seen that, an uncontrolled transmittable contacts between the infected and the susceptible can be catastrophic (high mortality rate), since no immunity and drugs at moment. This is evidence in the current outbreak, which has majorly affected the countries of Liberia, Sierra Leone and Guinnea and recorded the worstcase scenerio (highest dead rate, much more than the total of all death since the discovery of the virus in 1976). However, a better management option as revealed by the model is an aggressive quarantine system (with medical support), which will ultimately reduce the transmittable contacts, since by nature Ebola is highly infectious. Mathematical modelling is very essential in understanding and managing the outbreak of any disease. In the light of this work and its scope, where we assumed natural birth and death rates to be zero, i.e population $(\mathrm{N})$ to be constant, then we will recommend further research where there is a constant flow of new members into the whole population per unit time with infective or susceptible fractions. So $\mathrm{N}$ is no longer constant, it varies with time and even the rates; rates of infection, susceptibility etc become funtions of time.

\section{References}

[1] Wikipedia, "Ebola virus", http:// www.en.wikipedia.org/wiki/Ebola_virus, 2014.

[2] Z. Yarus, "A mathematical look at the Ebola virus", http://www.home2.fvcc.edu/ dhicketh/DiffEqns/Spring2012P rojects/Zach\%20Yarus\%20-

Final\%20Project/Final\%20Diffy\%20Q\%20project.pdf, 2012.

[3] J. Legrand, R.F. Grais, P.Y. Boelle, A.J. Valleron and A. Flahault, "Understanding the dynamics of Ebola epidemics", Epidemiology and Infection, $135^{\text {th }}$ ed., vol. 4, pp. 610-621. http://www.ncbi.nlm.nih.gov/pubmed/23007439, 2006.

[4] World Health Organization (WHO), "Ebola virus disease", http://www.who.int/mediacentre/factsheets/fs103/en/, 2014.

[5] Wikipedia, "Ebola virus epidemics in West Africa", http://www.en.m.wikipedia.org/wiki/Ebola virus epidemics $i$ n_West_Africa, 2015.

[6] C. Roy-Macaulay, "Ebola crisis triggers health emergency", http://www.dddmag.com/news/2014/07/ebola-crisis-triggershealth-emergency, 2014.

[7] T. Leslie. "Ebola: what is it and how does it spread?" $A B C$ News, http://www.abc.net.au/news/2014-07-30/ebola-virusexplainer/5635028, 2014.

[8] Wikipedia, "List of Ebola outbreaks", http://www.en.wikipedia.org/wiki/List of Ebola outbreaks, 2014.

[9] A. Nossiter, "Fear of Ebola breeds a terror of physicians", http://www.nytimes.com/2014/07/28/world/africa/ebolaepidemic-west-africa-guinea.html?r=0, 2014.

[10] D. Logan, A First Course in Differential Equations, Springer Science \& Business Media, New York, 2010.

[11] J.M. Mahaffy, "Epidemic models", http://www.rohan.sdsu.edu/ jmahaffy/courses/f09/math636/le" ctures/epidemics/epidemics.pdf, 2009.

[12] F. Brauer, J. Wu and P.V.D. Driessche, Mathematical Epidemiology, Springer-Verlag, Berlin Heidelberg, 2008.

[13] C. Yongli, Y. Kangb, W. Wang, and M. Zhaoc. "A Stochastic differential equation SIRS epidemic model with ratiodependent incidence rate", http://www.public.asu.edu/ ykang3/ les/CKWZ Stochastic.pdf, 2013.

[14] J. D. Murray, Mathematical Biology: 1. An Introduction, Springer Science \& Business Media, New York, 2002.

[15] S. G. Deo and V. Raghavendra, Ordinary Differencial Equations and Stability Theory, Tata Mc Graw-Hill Publishing Company Ltd, New York, 1980. 